\title{
Restrictions on pp scattering amplitude imposed by first diffraction minimum data obtained by TOTEM at LHC
}

\author{
V.N. Kovalenko, A.M. Puchkov, V.V. Vechernin, D.V. Diatchenko \\ Dep. of High Energy Physics, Saint-Petersburg State University, Russia; e-mail: nvkinf@rambler.ru
}

\begin{abstract}
We present the systematic analysis of the behavior of elastic pp scattering cross section in the region of the diffraction peak and the first diffraction minimum in the framework of quasi-eikonal GribovRegge approach, taking into account the unitarity condition. We use the formalism taking into consideration the dependence of pomeron signature factor on $t$. We show that although in this approach one can describe the general features of pp scattering, the behavior of pp elastic differential cross section in the vicinity of the first diffraction minimum measured by the TOTEM experiment at LHC can not be reproduced under any parameter values at standard assumptions of the model. Physically, in the quasi-eikonal Regge approach the proton proved to be "gray" and rather large, whereas the TOTEM data indicate that it is smaller and closer to "black" at LHC energies.
\end{abstract}

\section{INTRODUCTION}

Recently, fine experimental data on the behavior of elastic scattering cross section in the region of the diffraction peak and the first diffraction minimum was obtained by the TOTEM experiment for $7 \mathrm{TeV}$ pp collisions at the LHC [1,2]. The first restrictions on pp scattering amplitude imposed by this data were discussed in [3]. In the present work we consider the problem in the framework of quasi-eikonal Gribov-Regge approach 8, 9, taking into account the unitarity condition. We show that although in this approach one can describe the general features of the pp scattering such as the total elastic and inelastic cross sections, the slope of diffraction cone and the multiplicity density at midrapidities 10,11, nevertheless the behavior of pp elastic differential cross section in the vicinity of the first diffraction minimum measured by the TOTEM experiment at LHC [1,2] can not be reproduced under any parameter values at standard assumptions of the model.

\section{Quasi-eikonal Gribov-Regge approach}

The Gribov-Regge approach [8] is aimed to describe the high-energy hadronic interactions in the soft domain, when $s=\left(p_{1}+p_{2}\right)^{2} \rightarrow \infty$ and $t=\left(p_{1}-p_{1}^{\prime}\right)^{2} \lesssim m^{2}$. Here $p_{1}, p_{2}$ and $p_{1}^{\prime}$ are the four-momenta of colliding protons and the scattered hadron. For pp elastic interactions in the center mass system $(\mathrm{cms}): \sqrt{s}=2 E$ and $t=-k^{2}$, where $2 E$ and $k$ are the total energy and the transferred momentum in $\mathrm{cms}\left(k \approx\left|\mathbf{k}_{\perp}\right|\right.$ in this limit $)$. In this approach in the amplitude of elastic pp scattering at high energy the exchange by the reggeon with vacuum quantum numbers - the pomeron - is dominated. The amplitude corresponding to the one pomeron exchange is given by the following expression [8]:

$$
A_{1}(s, t)=G(t) D(s, t),
$$

where $D(s, t)$ is the pomeron propagator:

$$
D(s, t)=\eta(\alpha(t))\left(\frac{s}{s_{0}}\right)^{\alpha(t)-1} .
$$

Here $\alpha(t)$ is the pomeron trajectory:

$$
\alpha(t)=1+\Delta+\alpha^{\prime} t=1+\Delta-\alpha^{\prime} k^{2},
$$

and $\eta(\alpha(t))$ is the so-called signature factor:

$$
\begin{gathered}
\eta(\alpha(t))=-\frac{\exp \left(-\mathrm{i} \frac{\pi \alpha(t)}{2}\right)}{\sin \left(\frac{\pi \alpha(t)}{2}\right)}= \\
=\mathrm{i}+\operatorname{tg}\left(\frac{\pi}{2}(\alpha(t)-1)\right)=\mathrm{i}+\operatorname{tg}\left(\frac{\pi}{2}\left(\Delta-\alpha^{\prime} k^{2}\right)\right) .
\end{gathered}
$$

The $s_{0}$ is a parameter of the order of hadronic masses. Usually, one puts $s_{0}=1 \mathrm{GeV}^{2}$ and writes the pomeron propagator (2), introducing the whole rapidity width: $y \equiv \ln \left(s / s_{0}\right)$, in the following form:

$$
D(s, t)=\eta(\alpha(t)) \exp (\Delta y) \exp \left(-\alpha^{\prime} y k^{2}\right) .
$$

The factor $G(t)$ in (11) originates from the cut vertexes of pomeron coupling to protons. Usually, one 
approximates it by a Gaussian:

$$
G(t)=G_{0} \exp \left(-R^{2} k^{2}\right) .
$$

The Froissart bound at $s \rightarrow \infty$, applied to (1), leads to the restriction: $\alpha(0) \leq 1$, but the experimental data on the increase of the total cross sections and the multiplicity density at mid-rapidities with energy demands $\alpha(0)>1$, so one has to consider the supercritical pomeron with intercept $\alpha(0)-1=\Delta>0$. To meet the Froissart bound in this case one has to take into account the exchange by an arbitrary number of pomerons in the elastic pp amplitude:

$$
A(s, t)=\sum_{n=1}^{\infty} A_{n}(s, t),
$$

where $A_{n}(s, t)$ is the amplitude corresponding to the exchange by $n$ pomerons $[8$ :

$$
\begin{gathered}
A_{n}(s, t)= \\
=\frac{\mathrm{i}^{n-1}}{\pi^{n-1} n !} \int \prod_{i=1}^{n} d \mathbf{k}_{i \perp} D\left(s, \mathbf{k}_{i \perp}^{2}\right) G_{n}\left(\mathbf{k}_{1 \perp}, \ldots, \mathbf{k}_{n \perp}\right) \times \\
\times \delta^{(2)}\left(\sum_{i=1}^{n} \mathbf{k}_{i \perp}-\mathbf{k}_{\perp}\right) .
\end{gathered}
$$

Usually, one supposes the factorization of the cut vertex of $n$ pomerons coupling to proton:

$$
G_{n}\left(\mathbf{k}_{1 \perp}, \ldots, \mathbf{k}_{n \perp}\right)=C_{n} \prod_{i=1}^{n} G\left(\mathbf{k}_{i \perp}^{2}\right) .
$$

Assuming additionally

$$
C_{n}=C^{n-1},
$$

one comes to the quasi-eikonal Regge approximation for the pp amplitude.

\section{TAKING INTO ACCOUNT THE DEPENDENCE OF POMERON SIGNATURE FACTOR ON $t$}

Frequently, one replaces the signature factor (4) in the pomeron propagator (5) simply by i (see e.g. [12). In this approximation the amplitude of one pomeron exchange is pure imaginary. The present paper is aimed to investigate the behavior of the differential elastic pp cross section in the vicinity of first diffraction minimum at $-t=k_{\min }^{2}$, so we need to take into account the dependence of this factor on $t$. For this purpose we'll use the approximation suggested in [13]:

$$
\begin{aligned}
\eta(\alpha(t)) \approx & -\frac{\exp \left(-\mathrm{i} \frac{\pi \alpha(t)}{2}\right)}{\sin \left(\frac{\pi \alpha(0)}{2}\right)}=\eta(\alpha(0)) \exp \left(\mathrm{i} \frac{\pi}{2} \alpha^{\prime} k^{2}\right)= \\
& =\left[\mathrm{i}+\operatorname{tg}\left(\frac{\pi}{2} \Delta\right)\right] \exp \left(\mathrm{i} \frac{\pi}{2} \alpha^{\prime} k^{2}\right)
\end{aligned}
$$

which is valid when $|\Delta|,\left|\alpha^{\prime} k^{2}\right| \ll 1$ and hence $|\alpha(t)-1|=\left|\Delta-\alpha^{\prime} k^{2}\right| \ll 1$. The advantages of this approximation are that it has a Gaussian form and in the first order it coincides with the expansion of exact signature factor (4):

$$
\eta(\alpha(t)) \approx \mathrm{i}+\frac{\pi}{2} \Delta-\frac{\pi}{2} \alpha^{\prime} k^{2} .
$$

Note that in accordance with (21) and (4) the corrections affect only the real part of pomeron propagator and do not change its imaginary part.

The factorization suggestion, (9), and the Gaussian form of (6) and (11) enable to calculate the amplitudes $A_{n}(s, t)$, (8), explicitly:

$$
\begin{aligned}
& A_{n}(s, t)=\mathrm{i} C_{n}\left[1-\mathrm{i} \operatorname{tg}\left(\frac{\pi}{2} \Delta\right)\right]^{n} \frac{G_{0} \exp (\Delta y)}{n n !} \times \\
& \times\left(\frac{-G_{0} \exp (\Delta y)}{R^{2}+\alpha^{\prime} y^{\prime}}\right)^{n-1} \exp \left[-\frac{R^{2}+\alpha^{\prime} y^{\prime}}{n} k^{2}\right],
\end{aligned}
$$

where $y^{\prime}=y-\mathrm{i} \pi / 2$. Assuming additionally the quasi-eikonal approximation for $C_{n}$, (10), one has

$$
\begin{gathered}
A_{n}(s, t)=\mathrm{i}\left[1-\mathrm{i} \operatorname{tg}\left(\frac{\pi}{2} \Delta\right)\right]^{n} \frac{G_{0} \exp (\Delta y)}{n n !} \times \\
\times\left(\frac{-C G_{0} \exp (\Delta y)}{R^{2}+\alpha^{\prime} y^{\prime}}\right)^{n-1} \exp \left[-\frac{R^{2}+\alpha^{\prime} y^{\prime}}{n} k^{2}\right] .
\end{gathered}
$$

Now summing over $n$, (7), one can calculate the amplitude $A(s, t)$ and hence the elastic differential pp cross section:

$$
\frac{d \sigma_{e l}}{d t}=4 \pi|A(s, t)|^{2} .
$$

\section{Comparison With the EXPERIMENTAL DATa}

In paper [11] it is shown that the general features of the pp scattering at the LHC energy such as the total cross sections, the slope of diffraction cone and the multiplicity density at mid-rapidities can 
be reproduced in the quasi-eikonal Regge approach under the following values of parameters:

$$
\begin{gathered}
\Delta=0.19, \alpha^{\prime}=0.25 \mathrm{GeV}^{-2}, G_{0}=0.85 \mathrm{GeV}^{-2}, \\
R^{2}=3.3 \mathrm{GeV}^{-2}, C=1.8, \mu_{0}=1.5, \quad(16)
\end{gathered}
$$

where $\mu_{0}$ is the multiplicity at mid-rapidities from one cut pomeron. So with these values of parameters we calculate by (7) and (14) the elastic differential pp cross section, (15), as a function of cms transfer momentum and compare the obtained results with the corresponding experimental data, measured by the TOTEM experiment for $7 \mathrm{TeV}$ pp collisions at the LHC [1,2, The calculation were fulfilled with the signature factor both given by (11) and $\eta(\alpha(t))=\mathrm{i}$. The comparison is presented in Fig. 1. We see that although this set of parameters satisfactorily describes the total cross sections, the slope of diffraction cone, $B$, and the multiplicity density at mid-rapidities (see Table 1), nevertheless it can not reproduce the behavior of elastic differential pp cross section in a vicinity of the first diffraction minimum measured by the TOTEM experiment.

Note that the position of the minimum, $\left|t_{d i p}\right|=$ $k_{\text {min }}^{2} \approx 0.5 \mathrm{GeV}^{2}$, is in the domain where using of the approximation (11) are valid, since $\Delta=0.19 \ll 1$ and $\alpha^{\prime} k_{\text {min }}^{2} \approx 0.13 \ll 1$.

\begin{tabular}{|c|c|c|c|}
\hline & $\eta$, (11) & $\eta=\mathrm{i}$ & TOTEM data \\
\hline$\sigma_{\text {inel }}, \mathrm{mb}$ & 78.73 & 78.54 & $73.15 \pm 1.26$ \\
\hline$\sigma_{\text {tot }}, \mathrm{mb}$ & 98.86 & 97.56 & $98.58 \pm 2.23$ \\
\hline$\sigma_{e l}, \mathrm{mb}$ & 20.12 & 19.02 & $25.43 \pm 1.073$ \\
\hline$B, \mathrm{GeV}^{-2}$ & 23.53 & 23.41 & $19.9 \pm 0.3$ \\
\hline
\end{tabular}

Table 1: General characteristics (see the text) of pp scattering, calculated for parameters $C=1.8$ and $R^{2}=3.3 \mathrm{GeV}^{-2}$, in two versions of the model: with, (11), and without, $\eta=\mathrm{i}$, taking into account a dependence of the signature factor $\eta(\alpha(t))$, (4), on $t$, compared with the experimental data [1,2].

\begin{tabular}{|c|c|c|c|}
\hline & $\eta, \mathbf{1 1 1})$ & $\eta=\mathrm{i}$ & TOTEM data \\
\hline$\sigma_{\text {inel }}, \mathrm{mb}$ & 66.32 & 67.50 & $73.15 \pm 1.26$ \\
\hline$\sigma_{\text {tot }}, \mathrm{mb}$ & 104.07 & 102.99 & $98.58 \pm 2.23$ \\
\hline$\sigma_{\text {el }}, \mathrm{mb}$ & 37.75 & 35.49 & $25.43 \pm 1.073$ \\
\hline$B, \mathrm{GeV}^{-2}$ & 14.06 & 14.01 & $19.9 \pm 0.3$ \\
\hline
\end{tabular}

Table 2: The same as in Table 1, but calculated for parameters $C=1.0$ and $R^{2}=0.3 \mathrm{GeV}^{-2}$.

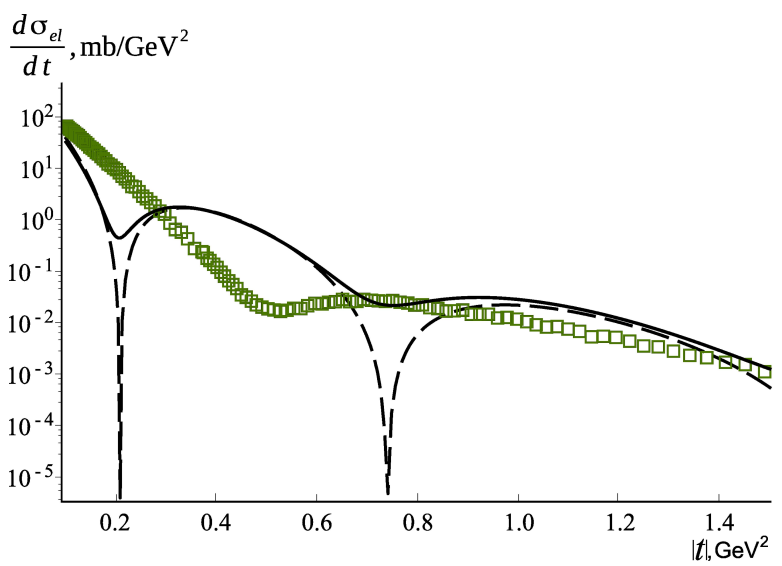

Figure 1: Elastic differential cross section of pp scattering, calculated for parameters $C=1.8$ and $R^{2}=3.3 \mathrm{GeV}^{-2}$, in two versions of the model: with, (11), (solid line) and without, $\eta=\mathrm{i}$, (dashed line) taking into account a dependence of the signature factor $\eta(\alpha(t))$, (4), on $t$. The points are the TOTEM experimental data 1,2].

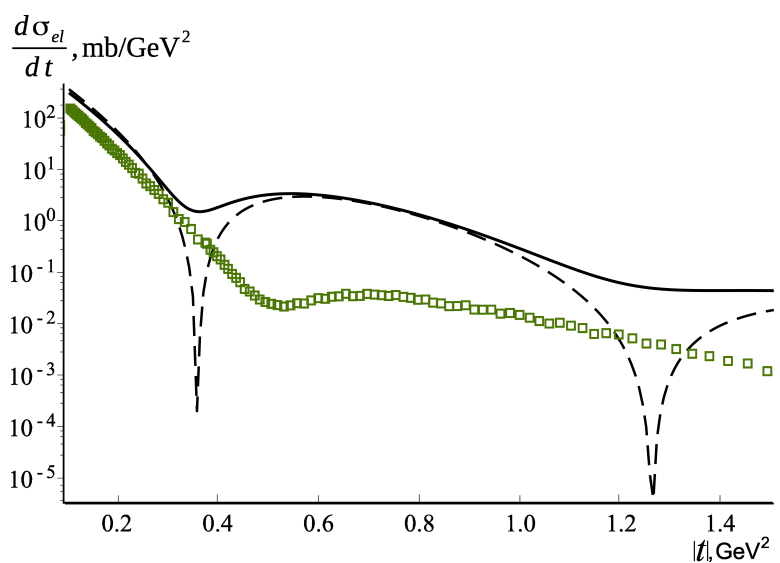

Figure 2: The same as in Fig. 1, but calculated for parameters $C=1.0$ and $R^{2}=0.3 \mathrm{GeV}^{-2}$.

Moreover, the following analysis shows that the TOTEM data can not be reproduced under any parameter values in the framework of the quasieikonal Regge approach under standard assumptions. Actually, by (14) we see that at given energy and fixed quasi-eikonal parameter $C$ we have only two effective parameters: $R^{2}+\alpha^{\prime} y$ and $G_{0} \exp (\Delta y)$. So we have calculated the $\sigma_{\text {tot }}$ and the position, $\left|t_{\text {dip }}\right|=k_{\text {min }}^{2}$, of the first diffraction minimum in $d \sigma_{e l} / d t$ varying independently the parameters $R^{2}$ and $G_{0}$. The other variables were fixed as follows: $\Delta=0.19, \alpha^{\prime}=0.25 \mathrm{GeV}^{-2}, C=1.8$. The results 


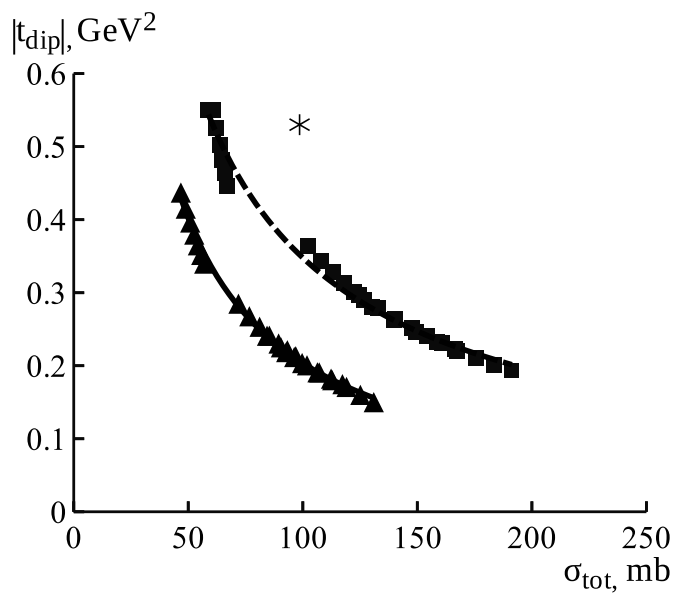

Figure 3: The position of the first diffraction minimum, $\left|t_{\text {dip }}\right|$, and the value of total cross section, $\sigma_{\text {tot }}$, calculated for parameters $\mathrm{C}=1.8$ (triangles) and $\mathrm{C}=1$ (squares), with different values of $R^{2}$ and $G_{0}$. The lines are approximations by the power law. The TOTEM experimental data is marked by an asterisk.

are presented in Fig. 3. We see that one can not describe simultaneously the total cross section and the position of the diffraction minimum in the framework of the model. All variation results are strongly correlated and lay along one curve, approximately described by the power law: $\left|t_{d i p}\right|=$ const $/ \sigma_{\text {tot }}$.

The situation is better in the simple eikonal approximation with $C=1$, but even in this case one can not obtain simultaneously the values $\sigma_{t o t}$ and $\left|t_{\text {dip }}\right|=k_{\text {min }}^{2}$ as in the TOTEM experiment, see Fig. 3. The general characteristics of pp scattering for this case are presented in Table 2 and Fig. 2

Physically one can understand this behaviour recalling that in the quasi-eikonal approach the impact parameter profile function $\gamma(s, b)$ of pp interaction (the Fourier transform of the $-2 \mathrm{i} A(s, t)$ ) has the form:

$$
\gamma(s, b)=C^{-1}[1-\exp (C \omega(s, b))],
$$

where $\omega(s, b))$ is the Fourier transform of the one pomeron exchange amplitude $-2 \mathrm{i} A_{1}(s, t)$, (11). At large energy in the model with supercritical pomeron at $b=0$ we have $\operatorname{Re} \omega(s, 0) \gg 1$ and hence $\gamma(s, 0) \approx 1 / C$. Taking this into account we see in Fig. 4 that in quasi-eikonal Regge approach at $C=1.8$ the nucleon proved to be "gray", $1 / C<1$, and rather large in the impact parameter space, which leads to smaller value of the first diffraction minimum position $\left(\approx 0.2 \mathrm{GeV}^{2}\right)$ in the momentum

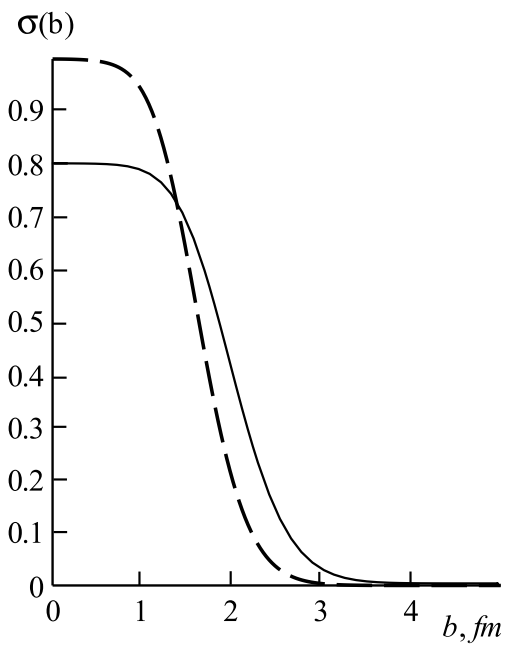

Figure 4: Profile function of pp collision $\sigma(b)$ calculated at $C=1.8$ (solid line) and $C=1$ (dashed line). $\quad \sigma(b)=2 \operatorname{Re}(\gamma(b))-|\gamma(b)|^{2}$, where $\gamma(b)$, (17), is a Fourier transform of the amplitude.

space, see Fig. 1. Whereas the TOTEM experiment by larger value of the first diffraction minimum position, $\left|t_{d i p}\right|=k_{\text {min }}^{2} \approx 0.5 \mathrm{GeV}^{2}$, indicates that the nucleon is smaller in the impact parameter space and closer to "black", what corresponds to better description with $C=1$ than with $C=1.8$, in Fig. 3.

The dependencies in Fig. 3 correspond to the fact that at given transparency, $C$, the increase of nucleon radius corresponds to the rise of total cross section and to the decrease of the Fourier transform width in momentum space. So by the comparison with the TOTEM data in Fig. 11 we see that since the data has the larger value of the first diffraction minimum position, than in quasi-eikonal Regge approach, then it corresponds to the smaller interaction radius and hence to more "black" nucleon to ensure the same total pp cross section.

This conclusion is in correspondence with the results, obtained by the modeling of the nucleon impact parameter profile function in 4] using the TOTEM data.

\section{Conclusions}

We come to the conclusion that in the framework of the quasi-eikonal Regge approach [8,9] under standard assumptions one can not describe the behavior of pp elastic differential cross section in the vicinity of the first diffraction minimum measured by 
the TOTEM experiment at LHC [1,2] under any parameter values, although one can describe the general features of the pp scattering such as the total elastic and inelastic cross sections, the slope of diffraction cone and the multiplicity density at mid-rapidities 10,11.

So to adjust the Regge model to the new experimental data we have to reconsider some standard simplifying assumptions of the model, namely, the factorization of the cut vertex of $n$ pomerons coupling to proton, (91), the quasi-eikonal assumption, (10), and the Gaussian form of the cut vertex of one pomeron coupling to proton, (6).

Most simply one can give up the latter assumption. This is also supported by the latest experimental data of the TOTEM collaboration [14. In the paper the authors argue that the first diffraction peak in pp scattering at $8 \mathrm{TeV}$ has the deviation from the simple Gaussian form, $\exp (B t)=$ $\exp \left(-B k^{2}\right)$, and is better described by the dependency $\exp \left(b_{1} t+b_{2} t^{2}\right)$ or even by $\exp \left(b_{1} t+b_{2} t^{2}+\right.$ $\left.b_{3} t^{3}\right)$. Clearly that we can reproduce this experimental feature in the framework of Regge approach if instead of the assumption on the Gaussian form of the cut vertex of one pomeron coupling to proton, (6), we will use one of the above non-Gaussian dependencies. Of course it will cause considerable additional computing difficulties, so we leave the realization of this program for future studies.

\section{ACKNowledgements}

The work was supported by the Saint-Petersburg State University grant 11.38.197.2014. V.K. acknowledges support of Special SPbSU Rector's Scholarship and Dynasty Foundation Scholarship, and V.V. has benefited from the RFFI grant 15-0202097.

\section{REFERENCES}

[1] Antchev, G., et al. (TOTEM Collaboration), 2011, Proton-proton elastic scattering at the LHC energy of $\sqrt{s}=7 \mathrm{TeV}$, Europhysics Letters 95, 41001, arXiv:1110.1385 [hep-ex].

[2] Antchev, G., et al. (TOTEM Collaboration), 2013, Measurement of proton-proton elastic scattering and total cross-section at $\sqrt{s}=7 \mathrm{TeV}$, Europhysics Letters 101, 21002.
[3] Dremin, I. M., 2014, Critical regime of proton elastic scattering at the LHC, JETP Letters 99, pp. 243-245, arXiv:1401.3106 [hep-ph].

[4] Dremin, I. M., 2014, Robust impact parameter profile of inelastic collisions, JETP Letters 100, pp. 491-493, arXiv:1407.4576 [hep-ph].

[5] Dremin, I. M., 2015, Interaction region of high energy protons, Physics-Uspekhi 58, 61, arXiv:1406.2153 [hep-ph].

[6] Dremin, I. M., 2015, Torus or black disk?, Bulletin of the Lebedev Physics Institute 42, pp. 21-25, arXiv:1404.4142 [hep-ph].

[7] Uzhinsky, V., Galoyan, A., 2012, Description of the Totem experimental data on elastic ppscattering at $\operatorname{sqrt}(\mathrm{s})=7 \mathrm{TeV}$ in the framework of unified systematic of elastic scattering data, arXiv:1111.4984v5 [hep-ph].

[8] Gribov, V. N., 1968, A Reggeon Diagram Technique, Soviet Physics JETP 26, pp. 414-423.

[9] Ter-Martirosyan, K. A., 1972, On the Reggeon graph scheme at available, not ultra-high, energies, Nuclear Physics B 36, pp. 566-574.

[10] Capella, A., Ferreiro, E. G., 2012, Charged multiplicities in pp and AA collisions at LHC, European Physical Journal C 72, 1936, arXiv:1110.6839v2 [hep-ph].

[11] Capella, A., Ferreiro, E. G., 2013, Protonproton multiplicity distributions at LHC and the Pomeron intercept, arXiv:1301.3339 [hep$\mathrm{ph}$.

[12] Werner, K., 1993, Strings, pomerons and the VENUS model of hadronic interactions at ultrarelativistic energies, Physics Reports 232, pp. 87-299.

[13] Volkovitskiy, P. E., Lapidus, A. M., Lisin, V. I., Ter-Martirosyan, K. A., 1976, Experimental Data FIT in the Theory of Pomeron with alpha-p (0) > 1 and Some of Its Consequences, Soviet Journal of Nuclear Physics 24, pp. 648-660.

[14] Antchev, G., et al. (TOTEM Collaboration), 2015, Evidence for non-exponential elastic protonproton differential cross-section at low $|\mathrm{t}|$ and $\sqrt{s}=8 \mathrm{TeV}$ by TOTEM, Nuclear Physics B 899, pp. 527-546, arXiv:1503.08111 [hep-ex]. 